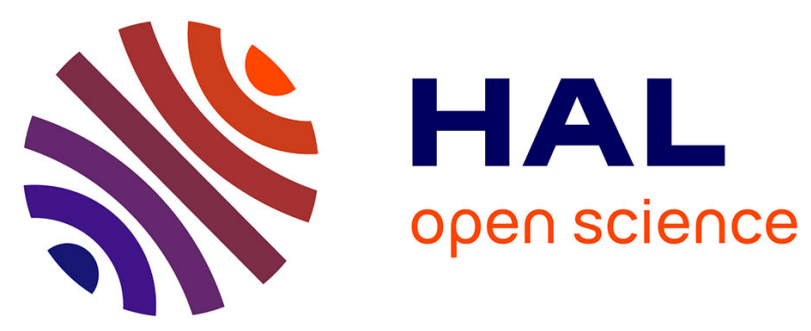

\title{
Reversible Strong Coupling in Silver Nanoparticle Arrays Using Photochromic Molecules
}

\author{
Anne-Laure Baudrion, Antoine Perron, Alessandro Veltri, Alexandre
}

Bouhelier, Pierre-Michel Adam, Renaud Bachelot

\section{To cite this version:}

Anne-Laure Baudrion, Antoine Perron, Alessandro Veltri, Alexandre Bouhelier, Pierre-Michel Adam, et al.. Reversible Strong Coupling in Silver Nanoparticle Arrays Using Photochromic Molecules. Nano Letters, 2012, 13 (1), pp.282-286. 10.1021/nl3040948 . hal-02370030

\section{HAL Id: hal-02370030 \\ https://hal-utt.archives-ouvertes.fr/hal-02370030}

Submitted on 27 Nov 2019

HAL is a multi-disciplinary open access archive for the deposit and dissemination of scientific research documents, whether they are published or not. The documents may come from teaching and research institutions in France or abroad, or from public or private research centers.
L'archive ouverte pluridisciplinaire HAL, est destinée au dépôt et à la diffusion de documents scientifiques de niveau recherche, publiés ou non, émanant des établissements d'enseignement et de recherche français ou étrangers, des laboratoires publics ou privés. 


\title{
Reversible strong coupling in silver nanoparticle
}

\section{arrays using photochromic molecules}

\author{
Anne-Laure Baudrion, ${ }^{*}{ }^{\dagger}$ Antoine Perron, ${ }^{\dagger}$ Alessandro Veltri, ${ }^{\ddagger}$ Alexandre \\ Bouhelier, ${ }^{\mathbb{I l}}$ Pierre-Michel Adam, ${ }^{\dagger}$ and Renaud Bachelot ${ }^{\dagger}$ \\ Laboratoire de Nanotechnologies et d'Instrumentation Optique, Institut Charles Delaunay UMR \\ CNRS 6279, Université de Technologie de Troyes, 12 Rue Marie Curie - CS 42060, 10004 Troyes \\ Cedex, France, Liquid Crystals Laboratory - INFM-CNR - CEMIF.CAL and Univ. Calabria \\ 87036 Arcavacata di Rende (CS), Italy, and Laboratoire Interdisciplinaire Carnot de Bourgogne \\ CNRS-UMR 6303 Université de Bourgogne 9, Avenue Alain Savary, BP 47870 F-21078 Dijon \\ Cedex \\ E-mail: anne_laure.baudrion@utt.fr
}

\begin{abstract}
In this letter, we demonstrate a reversible strong coupling regime between a dipolar surface plasmon resonance and a molecular excited state. This reversible state is experimentally observed on silver nanoparticle arrays embedded in a polymer film containing photochromic molecules. Extinction measurements reveal a clear Rabi splitting of $294 \mathrm{meV}$, corresponding to $\sim 13 \%$ of the molecular transition energy. We derived an analytical model to confirm our observations and we emphasize the importance of spectrally matching the polymer absorption
\end{abstract}

\footnotetext{
* To whom correspondence should be addressed

†Université de Technologie de Troyes

†Universita della Calabria

IUniversité de Bourgogne
} 
with the plasmonic resonance to observe coupled states. Finally, the reversibility of this coupling is illustrated by cycling the photochromic molecules between their two isomeric forms.

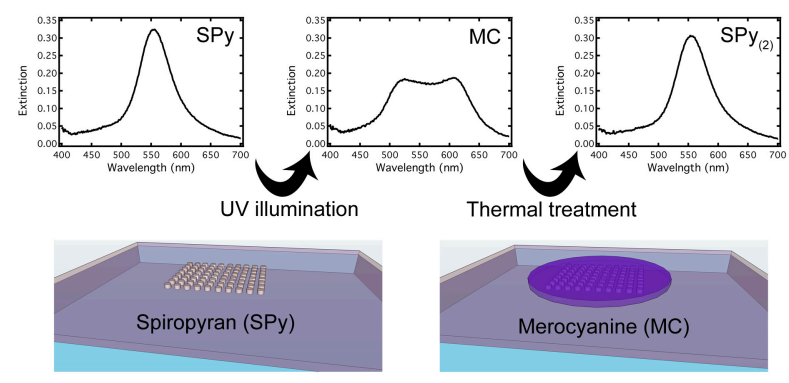

Keywords: Plasmon, spiropyran, exciton-plasmon coupling, Rabi splitting, active plasmonics

Hybrid plasmonic-molecular systems are a subject of great interest for their potential to control and engineer light-matter interactions at the nanoscale. In particular, surface plasmon modes can coherently hybridize with molecular excitons with large oscillator strength leading to a so-called strong coupling regime. Such regime and other coherent effects were reported in plasmonic cavityless experiments. ${ }^{1-5}$ Surface plasmon interaction with molecular states is at the basis of ultrafast functionalities that can be efficiently used to actively control molecular relaxation pathways ${ }^{6}$ and realize all-optical switches. ${ }^{7}$ In this context of controllable plasmonic devices, photochromic molecules like spiropyran compounds are of particular interest because they can undergo lightinduced reversible conformational changes associated with a large modification of the refractive index $n \cdot{ }^{8-11}$ Combined with the sensitivity of surface plasmon to the surrounding medium, this property was used to photo-modify the transmission characteristics of hole arrays, ${ }^{7}$ to realize plasmon waveguide modulators ${ }^{12}$ and to coherently couple to plasmonic nanocavities. ${ }^{13}$ In this letter, we report on the observation of an hybridization between the dipolar surface plasmon resonance of Ag nanoparticles and the electronic transition of spiropyran molecules. Specifically, we show that the coupling strength depends on the spectral position of the surface plasmon resonance and the conformational state of the molecules. This dependence provides an optical mechanism to ex- 
ternally control the hybridization state of the coupled system. We unambiguously illustrate such a command by cycling the photochromic transition of the molecules.

Silver nanoparticles were fabricated in large arrays using standard electron beam lithography on a glass substrate. The array periodicity was adjusted to keep constant the filling ratio $f$ defined as $f=4 \pi r^{2} / a^{2}$, with $r$ the particle radius and $a$ the pattern periodicity. The height of the nanoparticles was fixed at $50 \mathrm{~nm}$. Spiropyran 1',3'-Dihydro-8-methoxy-1',3',3'-trimethyl6-nitrospiro[2H-1-benzopyran-2,2'-(2H)-indole] molecules were diluted at 1.66 wt.-\% into $20 \mathrm{~g} / \mathrm{L}$ Polymethylmethacrylate (PMMA) solution in chlorobenzene. This solution was spin-coated onto the nanoparticle arrays to obtain a 60 nm-thick doped PMMA film. This specific photochromic molecule was chosen for its large yield of transition from the spiropyran (SPy) form to the merocyanine (MC) isomer when exposed to ultra-violet (UV) radiation. ${ }^{9}$ We verified that the PMMA film did not prevent the $\mathrm{SPy} \rightarrow \mathrm{MC}$ reaction. A film of doped polymer without $\mathrm{Ag}$ nanoparticles was exposed for $200 \mathrm{~s}$ onto the radiation from a xenon lamp filtered with a $400 \mathrm{~nm}$ low-pass filter. Figure 1(a) shows the absorbance spectra of the polymer before and after irradiation; the strong absorption peak at $570 \mathrm{~nm}$ confirms an isomerization to the MC state. ${ }^{14}$ We performed ellipsometric measurements to quantitatively estimate the wavelength dispersion of the optical constants $\Delta n$ and $\Delta k$ upon photoisomerization of the SPy-doped PMMA. Results are shown in Fig. 1(b). The variations of the refractive index and the extinction coefficient clearly show the molecular transition around $570 \mathrm{~nm}$ and are in good agreement with previous studies.$^{14,15}$

The surface plasmon resonances of the coated Ag nanoparticles were characterized by standard extinction spectroscopy. The arrays were illuminated by a halogen lamp and the transmitted light was collected by a $20 \times$ objective ( 0.4 numerical aperture) and sent to a fiber-coupled spectrometer. The probed region was estimated to be $53 \mu \mathrm{m}$ in diameter. All spectra obtained on the coated arrays where normalized by the transmission spectra of SPy-doped PMMA film without nanoparticles to eliminate the contribution of the molecular absorption band of the MC isomer. The solid black curves of Fig. 2(a), (b) and (c) display the normalized extinction spectra for 3 nanoparticle arrays covered by the SPy-doped PMMA. The diameter of the particles are $70 \mathrm{~nm}, 90 \mathrm{~nm}$ and $110 \mathrm{~nm}$, 

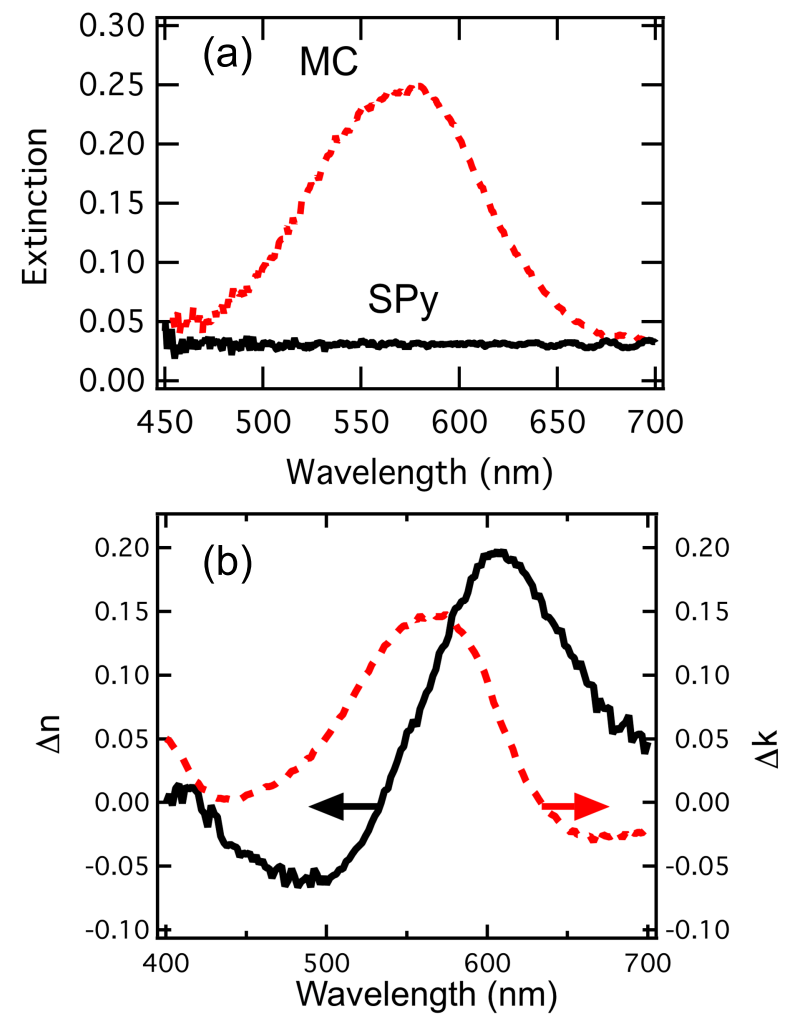

Figure 1: (a) Absorption spectra of the SPy doped PMMA thin layer before and after UV irradiation illustrating the conformational change of the spiropyran. (b) Ellipsometric measurements of the change of refractive index $\Delta n$ (solid black curve) and extinction coefficient $\Delta k$ (dashed red curve) between the two photochromic states. 
respectively. The vertical dashed line in each graph illustrates the spectral position of the molecular absorption peak of the MC form. The molecular transition is situated on the red side of the surface plasmon resonance for the $70 \mathrm{~nm}$-diameter particle array, close to the plasmonic resonance for the $90 \mathrm{~nm}$-diameter particle array and is on the blue side of the resonance for the $110 \mathrm{~nm}$-diameter particle array.

The dashed red curves in Fig. 2(a), (b) and (c) represent the normalized extinction spectra after UV exposure and isomerization of the spiropyran molecules. After exposition, the resonance positions of the $70 \mathrm{~nm}$ and the $110 \mathrm{~nm}$-diameter particle arrays are respectively blue and redshifted. A shoulder is also observed near the maximum absorption of the MC form. Each inset in Fig. 2 shows a fitted decomposition of the normalized extinction spectra after exposure by two spectrally separated Lorentzian peaks. The spectral variation of the surface plasmon resonances can be understood by using the ellipsometric measurements of Fig. 1(c). For example, the blueshift of the resonance for the array composed of $70 \mathrm{~nm}$ nanoparticles [Fig. 2(a)] can be related to the negative change of refractive index $\Delta n=-0.06$ at $\lambda=488 \mathrm{~nm}$. By differentiating the denominator of the polarizability $\alpha$ of a nanoparticle, ${ }^{16}$ we obtain $\Delta \lambda=-4 n \Delta n(d \varepsilon / d \lambda)^{-1}$. By taking $n=1.531$ at $\lambda=488 \mathrm{~nm}$ from the ellipsometric data and the dielectric function $\varepsilon$ of Ag from the literature, ${ }^{17}$ we obtain $\Delta \lambda=-8 \mathrm{~nm}$, which is very close to the $10 \mathrm{~nm}$ blue-shift measured in Fig. 2(a). This photochromic control of the surface plasmon resonance was already reported by Zheng et al. ${ }^{15}$ and was understood as an interaction in the weak coupling regime between the absorption band of the MC form and underlying plasmonic resonances.

The dispersion of the optical constants alone cannot explain the formation of two peaks in the extinction spectra of the $90 \mathrm{~nm}$-diameter particle array [Fig. 2(b)], as well as the formation of the shoulder in the other spectra. This spectral shape suggests an hybridization between two strongly interacting states. A Rabi splitting up to $700 \mathrm{meV}$ was previously observed in a multilayer plasmonic geometry ${ }^{13}$ where hybrid states were formed between an excitonic transition and the energy levels of a metal/Spy-doped PMMA/metal cavity. In our case, the two peaks in the extinction spectra of Fig. 2(b) are split by $294 \mathrm{meV}$, a separation corresponding to $\sim 13 \%$ of the molecular 

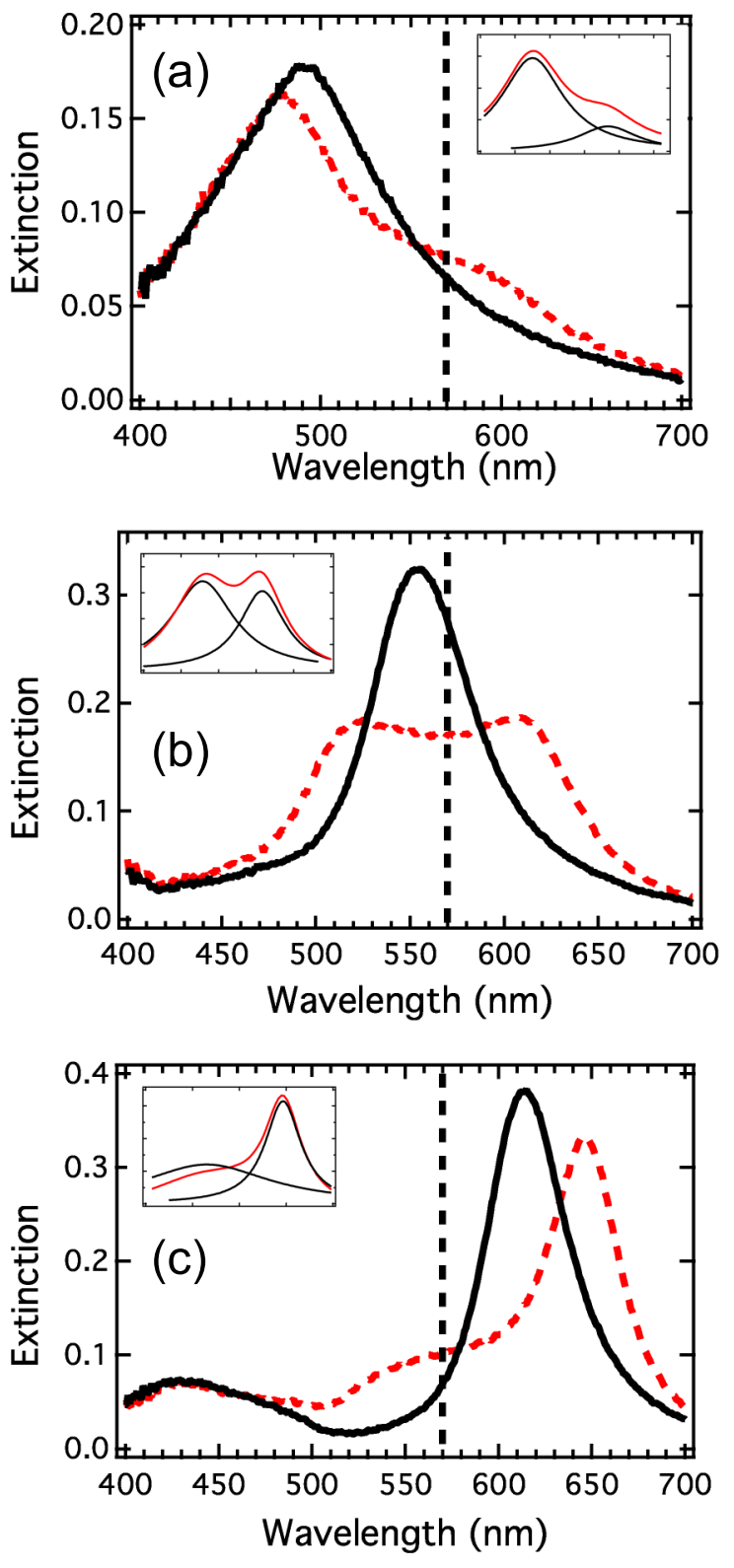

Figure 2: (a) to (c) Normalized extinction spectra of silver nanoparticle arrays embedded in a PMMA-doped SPy layer, before (black solid curve) an after (red dashed curve) UV irradiation. The diameter of the nanoparticles are respectively $70 \mathrm{~nm}, 90 \mathrm{~nm}$ and $110 \mathrm{~nm}$. The vertical dashed black lines correspond to the maximum absorbance of the MC layer. The insets represent the fitted decomposition of the red dashed curves by two separated Lorentzian peaks. 
transition energy. This value is smaller than the splitting ratio obtained for a multilayer cavity ${ }^{13}$ which yielded a $32 \%$ ratio. The lower quality factor of the dipolar resonance is at the origin of the reduced splitting ratio. Radiative decay, Joule losses and fabrication defects are responsible for a significant broadening of the surface plasmon resonance, thus limiting the interaction strength between the coupled states.

The passage from a weak to a strong coupling regime is affected by the spectral position of the plasmon resonance with respect to the molecular absorption band. For the smallest particles (70 $\mathrm{nm}$ in diameter), the plasmon resonance and the absorption peak are detuned by $80 \mathrm{~nm}$ and are therefore weakly interacting. However, a small shoulder on the red side of the resonance peak is the first indication of a hybridized state. For the $90 \mathrm{~nm}$-diameter particles, the detuning is only $14 \mathrm{~nm}$ and the signature of hybrid states is clearly observed in the normalized extinction spectra. Finally, the spectral response of the largest particles is detuned by $45 \mathrm{~nm}$ from the absorption peak of the MC form and a small shoulder on the blue side of the resonance peak is reminiscent of the hybridized state. This type of extinction spectroscopy analysis was performed for a series of diameter. The spectral position of each peak is plotted as a function of the diameter in Fig. 3 (a). A clear anti-crossing is observed when the plasmon resonances are nearly matched to the molecular excited states. The horizontal dashed line is the absorption maximum of the MC form, and the inclined dashed line shows the red-shift of the surface plasmon resonance with nanoparticle size before irradiation. For detuned resonances, only the SP signature is remaining since the molecular contribution is removed during the spectral re-normalization.

To support these experimental results, calculations were made using a model providing the extinction cross-section $\sigma$ of the nanoparticles embedded in an absorbing medium. ${ }^{18}$ Here, the absorbing medium was simulated using the experimental permittivity of the photo-merocyanine layer recorded by ellipsometry [Fig. 1(b)]. To account for the energy-shifting part of the retardation effect and the radiative loss which are missing in the model, expression 1 from Kuwata et al. ${ }^{19}$ was used to calculate the polarizability $\alpha$ of a nanoparticle: 

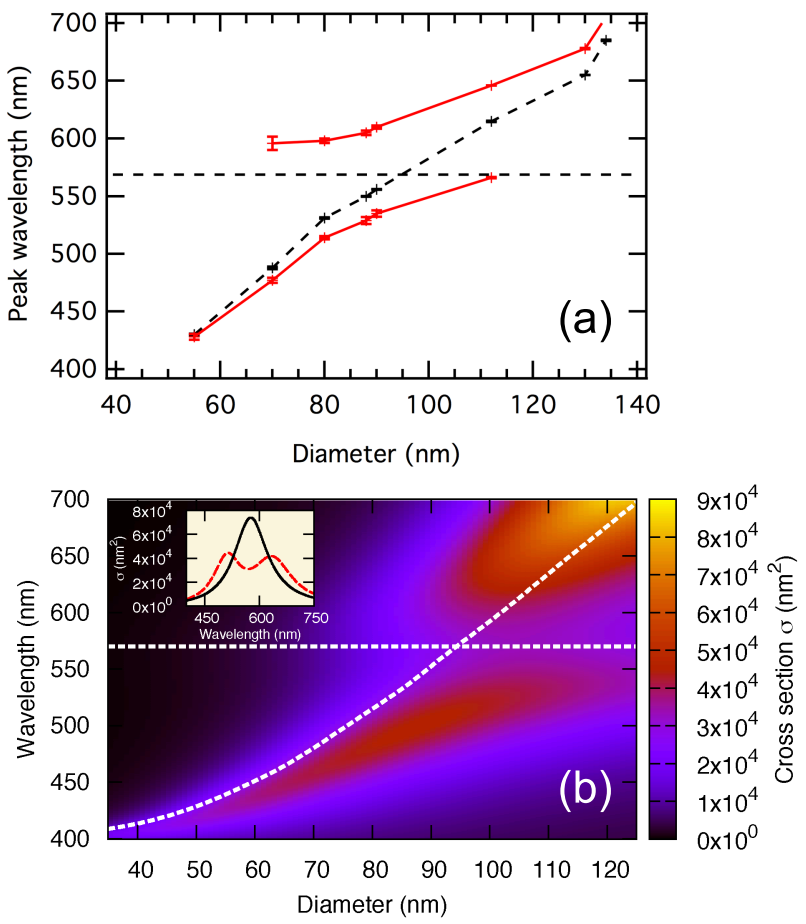

Figure 3: (a) Evolution of the peak wavelengths as a function of nanoparticle diameter. The dashed black lines represent the position of the MC absorption band (horizontal line) and the surface plasmon resonance of the arrays before irradiation. The red curve shows the evolution of the peaks after irradiation illustrating a anti-crossing of the dispersion curves. (b) Calculated map of the dispersion curves representing the extinction cross-section as a function of wavelength and nanoparticle diameter. The inset corresponds to a vertical cross-cut of the map where the splitting is maximum. The position of the plasmon resonance in the Spy-doped PMMA layer before the photochromic transition corresponds to the white dashed curve. The horizontal white dashed line corresponds to the maximum of the MC absorbance. 


$$
\alpha=4 \pi r^{3} \frac{1-0.1\left(\tilde{\varepsilon_{1}}+\tilde{\varepsilon_{2}}\right) \theta^{2} / 4}{\left(\tilde{\varepsilon_{1}}+2 \tilde{\varepsilon_{2}}\right) /\left(\tilde{\varepsilon_{1}}-\tilde{\varepsilon_{2}}\right)-\Xi_{1}\left(0.1 \tilde{\varepsilon_{1}}+\tilde{\varepsilon_{2}}\right) \theta^{2} / 4-\Xi_{2} \tilde{\varepsilon}_{2}^{2} \theta^{4}-i(2 / 3) \tilde{\varepsilon}_{2}^{3 / 2} \theta^{3}}
$$

where $\tilde{\varepsilon_{1}}$ and $\tilde{\varepsilon_{2}}$ are the complex refractive indices of the metal and the surrounding medium respectively, $\theta=2 \pi r \omega c, r$ is the particle radius and $\omega$ is the incident angular frequency. $\Xi_{1}=5.5$ and $\Xi_{2}=0.8$ are two phenomenological weighting factors introduced to obtain an optimal fit of the experimental results. The extinction cross-section was then calculated from the polarizability. ${ }^{16}$ The calculated cross-sections before and after irradiation are depicted in the inset of Fig. 3(b). The single resonance at $556 \mathrm{~nm}$ before isomerization of the SPy (solid black curve) is decomposed after the transition into two peaks at $513 \mathrm{~nm}$ and $633 \mathrm{~nm}$, corresponding to a splitting value of $400 \mathrm{meV}$. The small difference between this value and the experimental one $(294 \mathrm{meV})$ is probably due to the geometry differences between the modeled and the real systems. Indeed, the calculations deal with a spherical nanoparticle embedded in an homogeneous medium whereas the measured nanoparticles are rounded cylinders and coated with a finite layer, whose thickness corresponds approximately to the height of the nanoparticles. The calculated evolution of surface plasmon resonance of the nanoparticle embedded in MC-like medium is reported in Fig. 3 (b). The calculation confirms the presence of an anti-crossing of the dispersion curves when the surface plasmon resonance matches that of the molecular absorption band.

Finally, we show in the following that the hybridization is a controllable and a reversible process by cycling the spiropyran between its two isomeric forms. The thermal relaxation of the molecules in the SPy isomer is initiated by heating the irradiated merocyanine layer at $40^{\circ} \mathrm{C}$ during 10 minutes. After this treatment the SPy-doped-PMMA layer is again transparent in the visible range. Extinction spectra were performed during a complete isomerization cycle of the molecular film covering the $90 \mathrm{~nm}$ diameter particle array. Results are shown in Fig. 4. The extinction spectrum of the unexposed system displays a single surface plasmon peak (Fig. 4 (a)), whereas after UV irradiation the extinction spectrum displays again a splitting of the resonance indicative of a 
strong coupling regime discussed above (Fig. 4 (b)). After thermal treatment and a molecular conformational change, the extinction spectrum returns to the original state with only one peak visible (Fig. 4 (c)). Fatigue of the polymer is already visible after thermal treatment as the amplitude of the extinction peak is slightly lower in Fig. 4(c) compared to the one in Fig. 4(a).

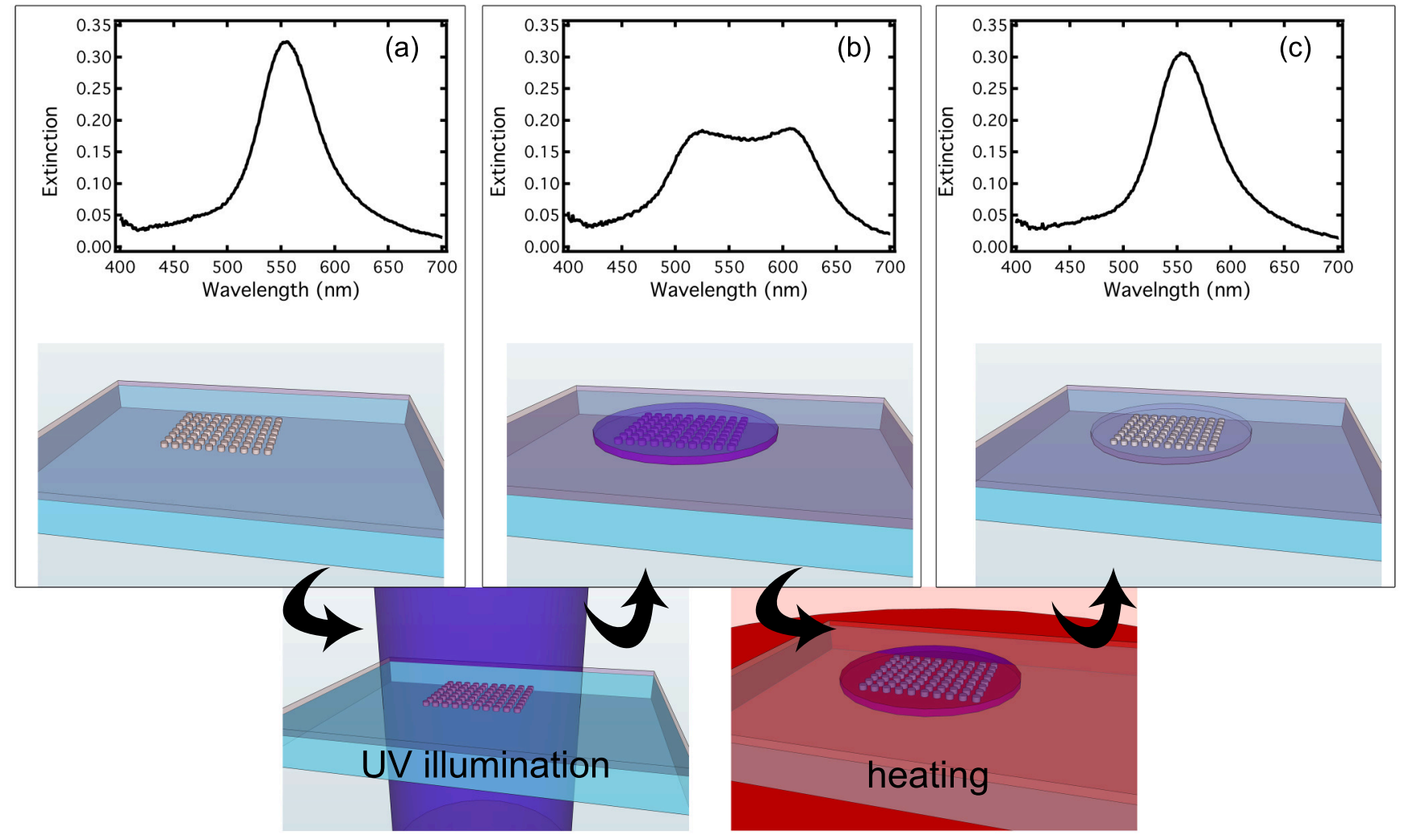

Figure 4: Extinction spectra of the $90 \mathrm{~nm}$-diameter particle array embedded in the photochromic layer for a complete isomeric cycle. (a) The spiropyran is in the Spy form. (b) The spiropyran is in the MC form after the UV illumination. (c) After a thermal treatment, the spiropyran is back in its Spy form.

In conclusion we observed a strong coupling between a dipolar surface plasmon and a molecular excited state. Such coupling is possible if the molecular transition and the plasmonic resonance are closely matched. Calculations are confirming the possibility to obtain a strongly coupled system with a splitting ratio in agreement with the experimental value. If the detuning between resonances is large, the surface plasmon response is determined by the refractive index change of the molecular layer. We further showed that the hybridized state between the surface plasmon and the electronic transition is reversible by acting on the conformational state of the photochromic 
molecule. Although demonstrated with an inherently slow thermal decoloration, the strong coupling regime could be controlled on a much faster time scale using a photo-activation of the merocyanine to spiropyran transformation.

Acknowledgements. The authors acknowledge the financial support of the Region ChampagneArdennes in the framework of scholarship for Ph.Ds. This work has been technically supported by Nano’Mat (www.nanomat.eu). A. B. gratefully acknowledges the support of the Regional Council of Burgundy under the PARI program.

\section{References}

(1) Pockrand, I. The Journal of chemical physics 1982, 77, 6289.

(2) Bellessa, J.; Bonnand, C.; Plenet, J. C.; Mugnier, J. Physical Review Letters 2004, 93, 36404.

(3) Wurtz, G. A.; Evans, P. R.; Hendren, W.; Atkinson, R.; Dickson, W.; Pollard, R. J.; Zayats, A. V.; Harrison, W.; Bower, C. Nano Letters 2007, 7, 1297-1303.

(4) Wiederrecht, G. P.; Wurtz, G. A.; Bouhelier, A. Chemical Physics Letters 2008,

(5) Bellessa, J.; Symonds, C.; Vynck, K.; Lemaitre, A.; Brioude, A.; Beaur, L.; Plenet, J.; Viste, P.; Felbacq, D.; Cambril, E.; Valvin, P. Physical Review B 2009, 80, 033303.

(6) Wiederrecht, G. P. G.; Hall, J. E. J.; Bouhelier, A. A. Physical Review Letters 2007, 98, 083001-083001.

(7) Dintinger, J.; Klein, S.; Ebbesen, T. W. Advanced Materials 2006, 18, 1267-1270.

(8) Berkovic, G.; Krongauz, V.; Weiss, V. Chemical Reviews 2000, 100, 1741-1754.

(9) Dürr, H.; Bouas-Laurent, H. Photochromism; Molecules and Systems; Elsevier Science, 2003.

(10) Schaudel, B.; Guermeur, C.; Sanchez, C.; Nakatani, K.; Delaire, J. A. Journal of Materials Chemistry 1997, 7, 61-65. 
(11) Tork, A.; Boudreault, F.; Roberge, M.; Ritcey, A. M.; Lessard, R. A.; Galstian, T. V. Applied optics 2001, 40, 1180.

(12) Pala, R. A.; Shimizu, K. T.; Melosh, N. A.; Brongersma, M. L. Nano Letters 2008, 8, 15061510.

(13) Schwartz, T.; Hutchison, J.; Genet, C.; Ebbesen, T. Physical Review Letters 2011, 106, 196405.

(14) Sasaki, K.; Nagamura, T. Applied Physics Letters 1997, 71, 434.

(15) Zheng, Y. B.; Kiraly, B.; Cheunkar, S.; Huang, T. J.; Weiss, P. S. Nano Letters 2011, 11, 2061-2065.

(16) Bohren, C. F.; Huffman, D. R. Absorption and Scattering of Light by Small Particles; John Wiley and Sons, Inc., New York, 1998.

(17) Palik, E. D. Handbook of Optical Constants of Solids; Academic Press, Orlando, 1985.

(18) Veltri, A.; Aradian, A. Physical Review B 2012, 85, 115429.

(19) Kuwata, H.; Tamaru, H.; Esumi, K.; Miyano, K. Applied Physics Letters 2003, 83, 4625. 